\title{
Acerca de la validez de las subespecies de Neotermes (Isoptera: Kalotermitidae) descritas de Chile
}

\author{
Concerning the validity of the subspecies of Neotermes (Isoptera: Kalotermitidae) \\ described for Chile \\ ARIEL CAMOUSSEIGHT ${ }^{1}$, ALEJANDRO VERA $^{2}$ \\ ${ }^{1}$ Museo Nacional de Historia Natural, Casilla 787, Santiago. E-mail: acamousseight@mnhn.cl \\ ${ }^{2}$ Universidad Metropolitana de Ciencias de la Educación, Casilla 474, Santiago. \\ E-mail: alveras2@hotmail.com
}

\begin{abstract}
The validity of the subspecies of Neotermes described by Goetsch (1) is discussed. Morphological and morphometric data were studied. The results showed that both subspecies correspond to the previously known species, Neotermes chilensis (2) and Porotermes quadricollis (3).
\end{abstract}

Key words: Insecta, Isoptera, Neotermes, Nomenclature, Chile.

\section{RESUMEN}

En el presente estudio se analiza la validez de las subespecies de Neotermes dadas para Chile por Goetsch (1). Se reevalúan los caracteres morfológicos y morfométricos que el mismo autor utiliza. Se determina que ambas subespecies corresponden respectivamente a especies previamente conocidas: Neotermes chilensis (2) y Porotermes quadricollis (3).

Palabras clave: Insecta, Isoptera, Neotermes, Nomenclatura, Chile.

\section{INTRODUCCION}

A pesar del exiguo número de especies de Isoptera presentes en Chile, un acertado reconocimiento de ellas no está exento de dificultades. Por una parte, Porotermes quadricollis (3) es claramente diferenciable $(4,5,6)$, pero, por otra, Neotermes chilensis (2) es confusa, fundamentalmente después del trabajo publicado por Goetsch (1). En este último se estableció la existencia de dos "variedades geográficas": Calotermes chilensis zapallarensis, de la región central del país, y Calotermes chilensis cayutuensis, de la región sur. Desconociendo, además, la existencia de Porotermes quadricollis, de acuerdo a lo señalado en el primer párrafo de su Introducción: "Hasta ahora en el territorio chileno con seguridad sólo se han observado dos especies de termitas: la Caloter- mes chilensis, en Chile continental, y la Calotermes gracilignathus, en la Isla de Juan Fernández".

De este modo, todas las posteriores listas y/o catálogos han debido incluir la especie $N$. chilensis y sus dos variedades $(7,8)$ o, en su defecto, las dos subespecies (9), en virtud del artículo 45.6.4. del International Code of Zoological Nomenclature (10). En este último caso la especie $N$. chilensis correspondería a la subespecie nominativa $N$. chilensis chilensis (2) en virtud del artículo 47.1 del mismo Código.

Sin embargo, empíricamente sólo dos especies de Isoptera nativas son reconocidas en las zonas centro y sur del país: $P$. quadricollis y $N$. chilensis sin subespecies. Enfrentados a esta dualidad teórica-práctica y con el objeto de aclarar la hipótesis planteada por Goetsch (1), en el presen- 
te trabajo se recrean las recolecciones de termitas en las zonas indicadas y se reestudian los caracteres en cada uno de los ejemplares obtenidos.

\section{MATERIAL Y METODOS}

Se estudió un total de 567 ejemplares distribuidos entre la IV y la X Regiones de Chile.

\section{CUADRO 1}

Ejemplares utilizados en los estudios morfológicos y morfométricos.

Specimens used for morphological and morphometrical studies.

\begin{tabular}{|c|c|c|c|}
\hline Región & $\mathrm{N}^{\mathrm{o}}$ ejem. & Datos de colecta & Especie \\
\hline \multirow[t]{2}{*}{ IV } & 31 & Punta Colorada, 16/07/2002. Leg. Camousseight, Vera & N.ch. $*$ \\
\hline & 53 & Los Vilos (en poste de madera), 02 y 05/2002. Leg. Vera & N.ch. \\
\hline $\mathrm{V}$ & 15 & Llolleo, 15/09/1972. Leg. Cruz & N.ch. \\
\hline \multirow[t]{11}{*}{ Metropolitana } & 103 & San Bernardo, 7/05/1997. & N.ch. \\
\hline & 3 & Santiago, 14/07/1992 & N.ch. \\
\hline & 15 & El Bosque, 13/02/1998 & P.q.** \\
\hline & 11 & Estación Central, 7/05/2000 & N.ch. \\
\hline & 4 & Santiago, $10 / 1996$ & N.ch. \\
\hline & 10 & Renca, 03/1998 & P.q. \\
\hline & 29 & Santiago, 21/01/1999. Leg. Blanco & N.ch. \\
\hline & 1 & Santiago, 1997 & N.ch. \\
\hline & 2 & Quinta Normal, 04/2002. Leg. Vera & N.ch. \\
\hline & 29 & Calera de Tango (en raulí muerto), 29/01/2002. Leg. Vera & N.ch. \\
\hline & 3 & La Obra, 14/08/2000. Leg. Vera & N.ch. \\
\hline VI & 34 & Lago Rapel, 1/03/1999 & P.q. \\
\hline VIII & 1 & Los Álamos, 24/07/1996. Leg. Cerda & P.q. \\
\hline VIII-IX & 1 & Cordillera de Nahuelbuta. & P.q. \\
\hline \multirow[t]{3}{*}{ IX } & 4 & Collipulli, 12/10/1962. Leg. Méndez & P.q. \\
\hline & 7 & Cautín, Lago Caburga, 02/2000. Leg. Vera & P.q. \\
\hline & 2 & Collipulli, 20/07/1992. Leg. Cerda & P.q. \\
\hline \multirow[t]{15}{*}{$X$} & 25 & Pto. Morror SW Pto. Montt, 12/05/1983. Leg. Merilov & P.q. \\
\hline & 19 & Pq. Nac. Puyehue, Anticura, 18-22/02/2001. Leg. Vera & P.q. \\
\hline & 2 & Pq. Nac. Puyehue, Anticura, 19-23/02/2002. Leg. Vera & P.q. \\
\hline & 14 & Llanquihue, Petrohué, 14-17/02/2001. Leg. Vera & P.q. \\
\hline & 6 & Arique, 01/2001. Leg. Nativo, Aguilar & P.q. \\
\hline & 9 & San José. Leg. Aguilar & P.q. \\
\hline & 6 & Lanco, 7/11/1998. Leg. Aguilar & P.q. \\
\hline & 2 & Lago Riñihue, 8/02/1958. Leg. Mujica. & P.q. \\
\hline & 2 & Río Lingue Km 8, 8/04/1969. Leg. Cekalovic & P.q. \\
\hline & 1 & Ancud, Chacao, 1/12/1969. Leg. Serra & P.q. \\
\hline & 1 & Petrohué, 13/10/1969. Leg. Solervicens & P.q. \\
\hline & 2 & Lago Rupanco, Osorno, 25/09/1968. Leg. Navarro & P.q. \\
\hline & 9 & $\begin{array}{l}\text { Pq. Nac. Pérez Rosales, Pta. Huano (en olivillo), 29/01/1973. } \\
\text { Leg. Solervicens }\end{array}$ & P.q. \\
\hline & 110 & $\begin{array}{l}\text { Pq. Nac. Alerce Andino (en canelo muerto), 14-18/02/2002. } \\
\text { Leg. Vera }\end{array}$ & P.q. \\
\hline & 1 & $\begin{array}{l}\text { Cayutué, Lago Esmeralda, Llanquihue, 14/10/1969. } \\
\text { Leg. Solervicens }\end{array}$ & P.q. \\
\hline
\end{tabular}

* N.ch.: Neotermes chilensis, ** P.q.: Porotermes quadricollis. 
Los individuos fueron clasificados según su casta: obrero, soldado e imago; en cada ejemplar se midieron la longitud de la cabeza y la talla corporal, considerando siempre las mandíbulas. En los imagos se midió, además, la talla incluyendo las alas, y se observó la presencia o ausencia de ocelos. En el caso de los obreros y soldados se registró la presencia o ausencia de ojos.

Particularmente en los soldados, se anotó la posición cruzada o no de las mandíbulas, se midieron el ancho máximo de la cabeza, longitud del clípeo-labro, además, el largo y ancho medio de la mandíbula izquierda. Todas las mediciones se efectuaron con micrómetro ocular en lupa binocular Bausch \& Lomb.
De acuerdo a los límites geográficos de las poblaciones del norte y del sur planteadas en el trabajo de Goetsch (1), los individuos estudiados se dividieron en 343 del norte (Regiones IV, V, Metropolitana y VI en su límite norte correspondiente al Lago Rapel) y 224 del sur (Regiones VIII en su límite sur, Los Alamos, IX y X).

Las mandíbulas de obreros y soldados se observaron en microscopio electrónico JEOL JSM25 SII Scanning Microscope; las fotografías fueron obtenidas con cámara Mamiya 6x7.

Para fines de distribución se consideraron, además, 404 ejemplares.

La totalidad del material estudiado queda depositada en la Colección del Museo Nacional de Historia Natural.

\section{CUADRO 2}

Ejemplares adicionales para el establecimiento de la distribucion geográfica de las especies. Supplementary specimens to establish geographical distribution of the species.

\begin{tabular}{|c|c|c|c|}
\hline Región & $\mathrm{N}^{\mathrm{o}}$ ejem. & Datos de colecta & Especie \\
\hline III & $8 *$ & Pto. Huasco. 09/2002. Leg. S.A.G. & P.q. \\
\hline \multirow[t]{2}{*}{ IV } & 49 & Guanaqueros, 18/01/2003. Leg. Elgueta & N.ch. \\
\hline & 1 & P. N. Fray Jorge. 29/07/1970. Leg. Solervicens & N.ch. \\
\hline \multirow[t]{6}{*}{$\mathrm{V}$} & 1 & Valparaíso, 03/1983. Leg. Prado & N.ch. \\
\hline & 120 & Las Chilcas, 11/08/2002.(en quillay). Leg. Vera & N.ch. \\
\hline & 4 & Valparaíso, 12/1993. Leg. Unanue. & N.ch. \\
\hline & 8 & Villa Alemana, 20/07/1962. Leg. Solervicens. & N.ch. \\
\hline & $2 *$ & Valparaíso, 3/1983. (en caja de embalaje). Leg. Prado & P.q. \\
\hline & 1 & Rocas de Sto. Domingo, 3/02/1970. Leg. Millas. & P.q. \\
\hline \multirow[t]{3}{*}{ Metropolitana } & 6 & Santiago, Caleu, 22/08/1987. Leg. Cerda & N.ch. \\
\hline & 2 & Santiago, 01-02/1982 & P.q. \\
\hline & 2 & Rinconada de Maipú, 29/4/2002. Leg. Mondaca & N.ch. \\
\hline \multirow[t]{2}{*}{ VI } & 3 & Santa Cruz, 26/07/2000 & N.ch. \\
\hline & 3 & Las Cabras, 10-23/12/1954. & P.q. \\
\hline \multirow[t]{2}{*}{ VII } & 29 & Vilches Altos, 1-3/02/2002. Leg. Vera & P.q. \\
\hline & 4 & Vilches Altos, 13/09/1998. Leg. Vera & P.q. \\
\hline VIII & 2 & Chillán, P. Marchant, 14/01/1989. Leg. Roitman & P.q. \\
\hline \multirow[t]{2}{*}{ IX } & 3 & Lican Ray, 22/01/1992. Leg. Cerda & P.q. \\
\hline & 6 & Cuesta Lastarria, 23/09/1968. Leg. Martínez. & P.q. \\
\hline \multirow[t]{5}{*}{$\mathrm{X}$} & 13 & Valdivia, Riñihue, 7/12/1966. Leg. Collarte & P.q. \\
\hline & 5 & Valdivia, Llancacura, 17/01/1966. Leg. Aravena & P.q. \\
\hline & 2 & Osorno, Bahía Mansa, 26/09/1968. Leg. González. & P.q. \\
\hline & 120 & Chiloé, Cucao, 30/01/2003. (en tronco de tepa). Leg. Vera & P.q. \\
\hline & 10 & $\begin{array}{l}\text { P. N. Vicente Pérez Rosales, Pta. Huano. 26/01/1973. } \\
\text { Leg. Solervicens. }\end{array}$ & P.q. \\
\hline
\end{tabular}

* Registros en puertos no considerados para distribución geográfica. 


\section{RESULTADOS Y DISCUSION}

Análisis de las variables morfométricas. La diferencia de talla promedio de los obreros del norte y del sur es estadísticamente significativa ( $\mathrm{P}<$ 0,01); a pesar de ello, no puede ser considerado un carácter diagnóstico, puesto que los rangos de ambos grupos se superponen, los del norte van de 7,44-10,16 mm y los del sur de 6,19-7,81 mm; debido probablemente a dos factores: la gran flexibilidad del tegumento y no discriminar entre individuos de diferentes instars de desarrollo. Por su parte, el largo de la cabeza no alcanza significación estadística $(P>0,05)$, manteniendo, levemente, la tendencia observada para la talla.

\section{CUADRO 3}

Promedio de talla y largo cabeza para obreros. Size and head length averages of workers.

\begin{tabular}{|lll|}
\hline & Talla $\mathrm{mm}$ & Largo cabeza $\mathrm{mm}$ \\
\hline \multirow{2}{*}{ Norte } & $\mathrm{N}=200$ & $\mathrm{~N}=200$ \\
& $\mathrm{X}=8,8 \pm 1,36$ & $\mathrm{X}=2,0 \pm 0,15$ \\
\multirow{2}{*}{ Sur } & $\mathrm{N}=141$ & $\mathrm{~N}=141$ \\
& $\mathrm{X}=7,0 \pm 0,81$ & $\mathrm{X}=1,9 \pm 0,23$ \\
\multirow{2}{*}{ t Student } & $\mathrm{P}<0,01$ & $\mathrm{P}>0,05$ \\
\hline
\end{tabular}

Los resultados así obtenidos no permiten diferenciar los grupos, de igual forma como Goetsch (1), al parecer, considerara, puesto que no comenta las medidas dadas en su trabajo.

Respecto de los soldados, se puede observar que los grupos del norte y del sur no tienen dife- rencias estadísticamente significativas entre los promedios de las tallas $(\mathrm{P}>0,05)$, pero sí respecto de la longitud de las cabezas $(\mathrm{P}<0,01)$. A pesar de ello, el carácter no es diferencial, porque el rango de los del sur $(4,46-5,54 \mathrm{~mm})$ está contenido en los del norte $(5,03-6,17)$. Luego, talla y largo de cabeza no servirían como caracteres de diagnóstico en la casta de soldados, de igual forma como se ha demostrado para los obreros.

Goetsch (1) describe o señala en esquemas otras tres diferencias respecto de los soldados: mandíbulas más delgadas en los individuos del norte, quienes, además, las conservan abiertas después de muertos, a diferencia de los del sur que las conservarían cerradas o cruzadas y la longitud del clípeo-labro sería mayor en los individuos del sur.

Del análisis de 73 soldados, se establece que conservar las mandíbulas abiertas o cerradas después de muertos no sería representativo de ningún grupo en particular.

Respecto del grosor de las mandíbulas, los individuos del norte las tendrían en promedio ( $\mathrm{P}<0,01)$ más delgadas. Sus rangos fluctúan entre 4,31-5,69 mm, en tanto que los del sur, entre 3,10-3,90 mm. Y la longitud clípeo-labro sería en promedio $(\mathrm{P}<0,01)$ menor para los del norte.

En cuanto a la talla de los imagos, a pesar que los promedios de los grupos del norte y del sur son estadísticamente significativos $(\mathrm{P}<0,01)$, no es posible discriminar si un individuo pertenece a uno u otro grupo, dado el amplio recubrimiento de los rangos. El argumento se revierte al considerar el largo total con alas, que sí permitiría diferenciar a los individuos del norte con una talla

\section{CUADRO 4}

Promedios de talla, largo cabeza, largo/ancho mandíbula izquierda y largo clípeo-labro para soldados. Size, head length, ratio length/width of left mandible and length of clypeus-labrum of soldiers.

\begin{tabular}{|lllll|}
\hline & Talla mm & Largo cabeza $\mathrm{mm}$ & $\begin{array}{l}\text { Largo/ancho } \\
\text { mandíbula } \\
\text { izquierda } \mathrm{mm}\end{array}$ & $\begin{array}{l}\text { Largo Clípeo-labro } \\
\mathrm{mm}\end{array}$ \\
\hline Norte & $\mathrm{N}=54$ & $\mathrm{~N}=54$ & $\mathrm{~N}=52$ & $\mathrm{~N}=54$ \\
Sur & $\mathrm{X}=11,5 \pm 1,19$ & $\mathrm{X}=5,6 \pm 0,57$ & $\mathrm{X}=5,0 \pm 0,69$ & $\mathrm{X}=0,47 \pm 0,12$ \\
& $\mathrm{~N}=27$ & $\mathrm{~N}=28$ & $\mathrm{~N}=26$ & $\mathrm{~N}=28$ \\
t Student & $\mathrm{X}=11,2 \pm 0,96$ & $\mathrm{X}=5,0 \pm 0,54$ & $\mathrm{X}=3,5 \pm 0,40$ & $\mathrm{X}=0,58 \pm 0,11$ \\
& $\mathrm{P}>0,05$ & $\mathrm{P}<0,01$ & $\mathrm{P}<0,01$ & $\mathrm{P}<0,01$ \\
\hline
\end{tabular}




\section{CUADRO 5}

Promedios de talla de imagos con o sin alas.

Mean length of adult with and without wings.

\begin{tabular}{|lll|}
\hline & Largo sin alas mm & Largo con alas mm \\
\hline \multirow{2}{*}{ Norte } & $\mathrm{N}=89$ & $\mathrm{~N}=69$ \\
& $\mathrm{X}=9,0 \pm 1,03$ & $\mathrm{X}=20,5 \pm 0,69$ \\
Sur & $\mathrm{N}=55$ & $\mathrm{~N}=44$ \\
& $\mathrm{X}=11,0 \pm 1,25$ & $\mathrm{X}=24,1 \pm 0,86$ \\
t Student & $\mathrm{P}<0,01$ & $\mathrm{P}<0,01$ \\
\hline
\end{tabular}

promedio más pequeña, entre 19,8-21,2 mm, y los del sur de mayor talla, entre 23,24-24,96 mm.

En resumen, las variables morfométricas de las poblaciones del norte se caracterizarían por soldados con mandíbulas delgadas, clípeo-labro corto e imagos de largo con alas entre 19,8-21,2 $\mathrm{mm}$, en contraposición con los del sur, con mandíbulas gruesas, clípeo-labro largo e imagos de largo con alas mayores entre 23,24-24,96 mm.

Análisis de las variables morfológicas. En soldados adultos, en obreros adultos o ninfas de diferentes instars de desarrollo, la presencia de ojos desarrollados o rudimentarios no sería del $100 \%$.

La ausencia de ojos en algunos obreros podría ser explicada como consecuencia de un menor desarrollo postembrionario, pero no en el caso de los soldados, cuyo estado es terminal.

Presencia de ocelos en los imagos es un carácter diagnóstico de la Familia Kalotermitidae (11), por lo tanto, el 67,0\% de los individuos del norte y la totalidad de los del sur no pertenecerían a esta familia y, por lo mismo, al género Neoter- mes. En tanto que la Familia Termopsidae Grassé, 1949 (12) tiene como carácter diagnóstico la ausencia de ocelos y, en consecuencia, lo mismo ocurriría con el único género presente en Chile de esta familia: Porotermes Hagen, 1858 (13).

Por lo anterior, pensamos que Goetsch (1), por desconocimiento de la especie $P$. quadricollis, habría incurrido en la inadecuada subdivisión de la especie Neotermes chilensis, y, en consecuencia, su subespecie Calotermes chilensis cayutuensis sería sinónimo de $P$. quadricollis.

En resumen, las variables morfológicas, el desarrollo de ojos en obreros y soldados no son constantes, independientemente de su pertenencia a grupos del norte o del sur. Mientras que la presencia de ocelos en los adultos es claramente un carácter diagnóstico entre otros, habría permitido la erección de la Familia Kalotermitidae. Por lo tanto, los imagos pertenecientes al grupo del norte encierran dos entidades taxonómicas, indiferenciables por estudios morfométricos.

Síntesis del análisis de variables. Tanto obreros e imagos en los grupos del norte y del sur resultaron indistinguibles por las variables de largo de cabeza y/o talla. Estos mismos individuos, al ser analizados respecto de la morfología mandibular, permiten establecer que obreros e imagos del norte pertenecen a la Familia Kalotermitidae, con mandíbula derecha caracterizada por un diente apical, dos marginales y lámina molar, esta última con una longitud casi igual a la del margen posterior del segundo diente marginal (11) (figura 1). En tanto que los del sur presentan en su mandíbula derecha un conspicuo pequeño diente subsidiario en la base del extremo anterior del primer diente marginal $(4,5,6)$ (figura 2), característico de la Familia Termopsidae.

\section{CUADRO 6}

Presencia o ausencia de ojos en soldados, obreros e imagos.

Presence or absence of eyes in soldiers, workers and adult alates.

\begin{tabular}{|lccccccc|}
\hline & \multicolumn{3}{c}{ Norte } & & \multicolumn{3}{c|}{ Sur } \\
\cline { 2 - 3 } \cline { 7 - 8 } \cline { 6 - 8 } & $\mathrm{N}^{\circ}$ Individuos & Presencia & Ausencia & & $\mathrm{N}^{\circ}$ individuos & Presencia & Ausencia \\
\hline Soldados & 54 & $98,2 \%$ & $1,8 \%$ & & 28 & $78,6 \%$ & $21,4 \%$ \\
Obreros & 200 & $99,5 \%$ & $0,5 \%$ & & 141 & $81,6 \%$ & $18,4 \%$ \\
Imagos & 88 & $33,0 \%$ & $67,0 \%$ & & 55 & $0 \%$ & $100 \%$ \\
\hline
\end{tabular}




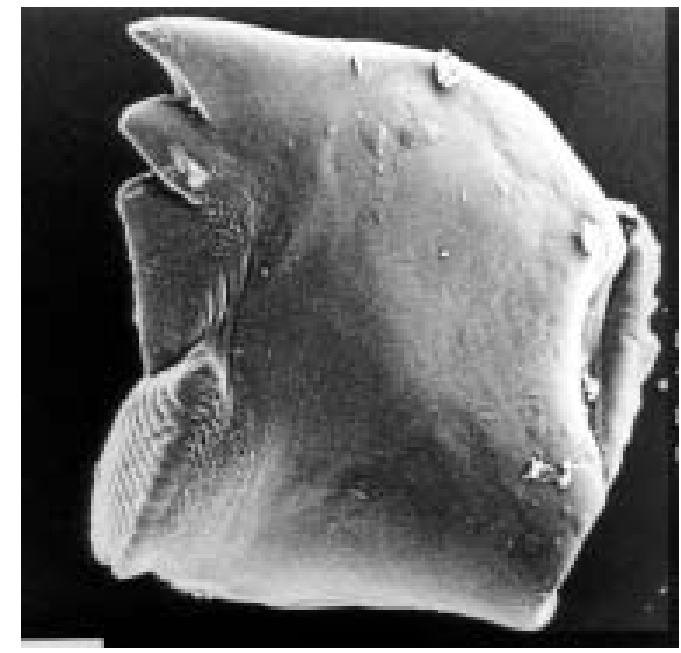

Figura 1. Mandíbula derecha de Neotermes chilensis (obrero e imago).

Right mandible of Neotermes chilensis (workers \& adults).

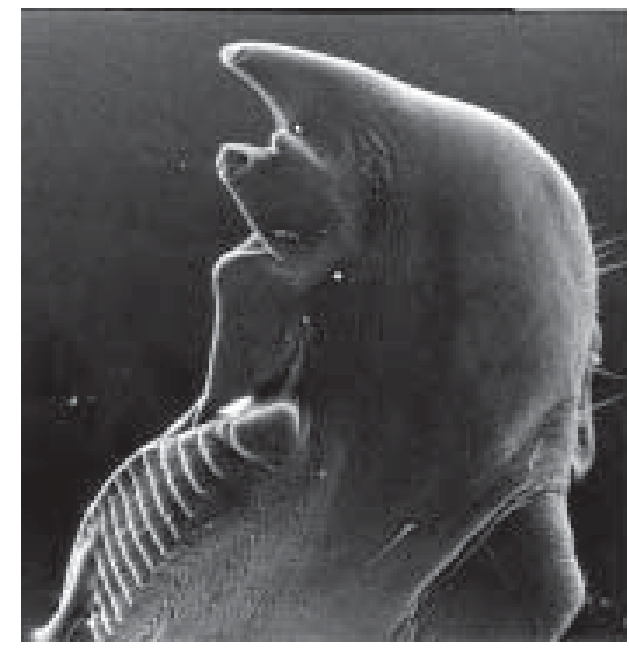

Figura 2. Mandíbula derecha de Porotermes quadricollis (obrero e imago).

Right mandible of Porotermes quadricollis (workers \& adults).

Al reevaluar en los imagos el estudio del carácter largo total con alas, entre los individuos del norte con presencia de ocelos versus los individuos del norte y sur con ausencia de los mismos, se obtuvo para sus promedios una $\mathrm{P}<0,01$ de acuerdo al t Student, reafirmando la validez estadística respecto de la mayor talla, considerada las alas, para los ejemplares del sur. Sin embargo, los rangos de talla constituyen prácticamente un continuo: Neotermes 19,38-20,42 mm ( $=24)$, Poro- termes $20,59-24,31 \mathrm{~mm}(\mathrm{~N}=89)$, por lo tanto, el largo con alas no podría ser considerado como un carácter diagnóstico.

Respecto de los soldados, y como se demostró en el análisis morfométrico, los del norte tendrían un clípeo-labro más corto y mandíbulas más delgadas (figura 3), en tanto que los del sur presentarían comparativamente un clípeo-labro más largo y mandíbulas más gruesas (figura 4).

Finalmente la evaluación realizada respecto de la presencia o ausencia de ojos permite señalar que ésta es una condición variable tanto en obreros como en soldados, independientemente de la especie que se trate, lo cual contradice lo afirma-

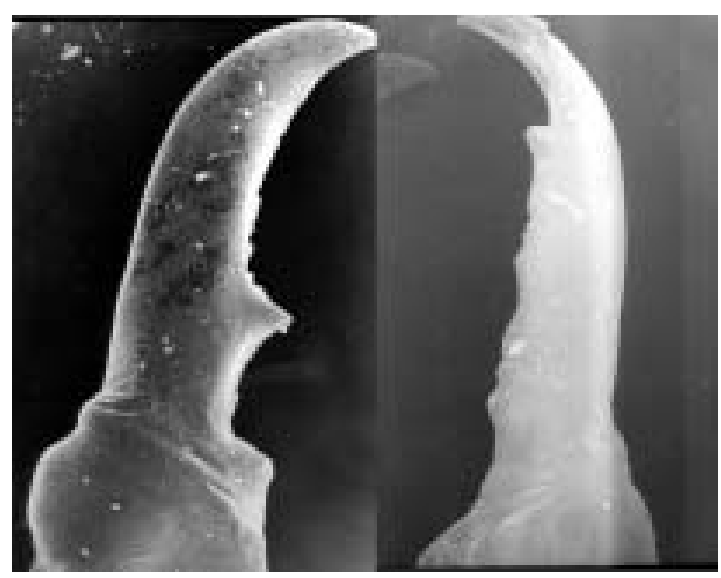

Figura 3. Mandíbulas soldado Neotermes chilensis Mandibles of soldiers of Neotermes chilensis.

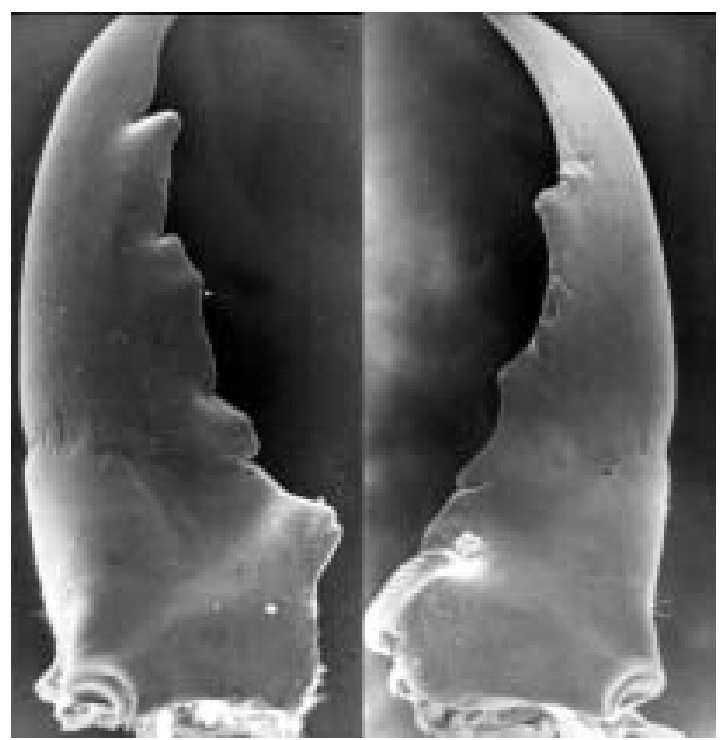

Figura 4. Mandíbulas soldado Porotermes quadricollis. Mandibles of soldiers of Porotermes quadricollis. 
BOSQUE 26(2): 5-21, 2005

Subespecies de Neotermes descritas de Chile

do por una parte por Goetsch (1), quien señala que todos los obreros presentan pequeños ojos y, por otra, Villan (6), quien indica que los soldados de Porotermes no presentan ojos.

Los 971 ejemplares considerados en el presente trabajo fueron identificados indicándose en las tablas de distribución (cuadros 1 y 2). De acuerdo con estos resultados, Neotermes chilensis se distribuiría entre la IV Región: Punta Colorada (29³9' lat.) y la VI Región: Santa Cruz (34 39' lat.). En tanto que Porotermes quadricollis lo haría entre la Región Metropolitana: Santiago ( $33^{\circ} 37^{\prime}$ lat.) y la X Región: Cucao (42 $38^{\circ}$ lat.) (figura 5).

La muestra de la III Región: Puerto Huasco, efectuada por funcionarios del Servicio Agrícola y Ganadero, y la del puerto de Valparaíso, en caja de embalaje, no son consideradas en la distribución de la especie, por tratarse de situaciones accidentales que no son indicadoras de una población establecida, como ha sido el caso de otras especies dadas como presentes en el país, ejemplo de ella es Coptotermes testaceus (Linneo) (7)

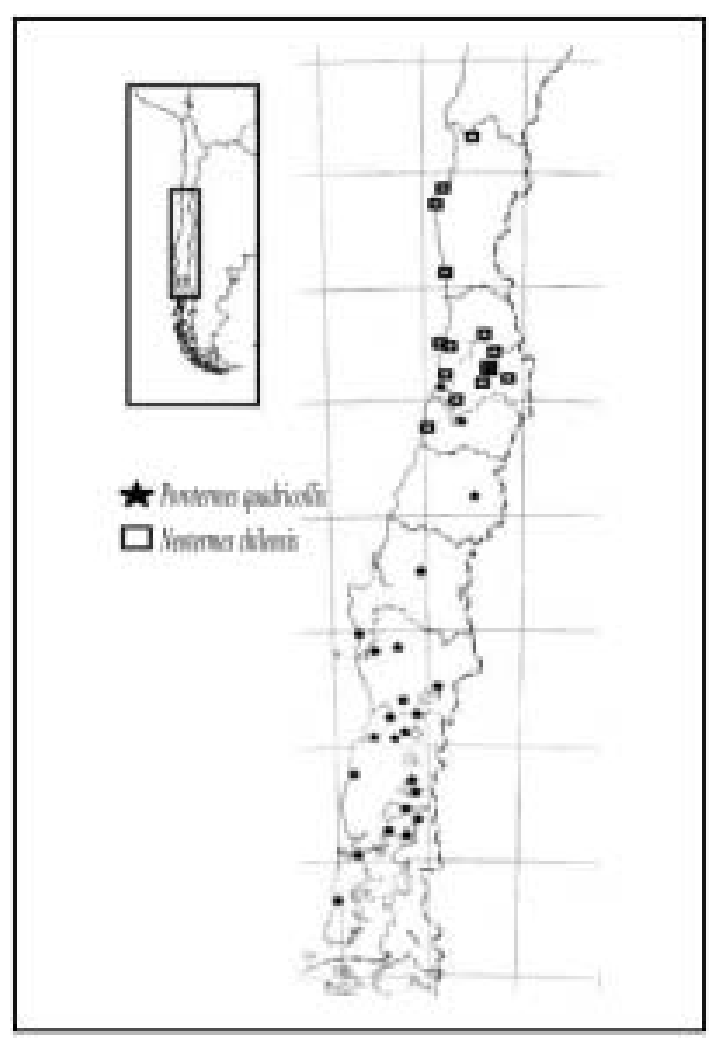

Figura 5. Distribución geográfica de Neotermes chilensis y Porotermes quadricollis.

Geographical distribution of Neotermes chilensis and Porotermes quadricollis. por hallazgos en el puerto de Valparaíso y que nunca más han sido encontradas.

\section{CONCLUSIONES}

La subespecie Calotermes chilensis cayutuensis Goetsch, 1933, es sinónimo de la especie Porotermes quadricollis (Rambur, 1842).

La subespecie nominal Calotermes chilensis zapallarensis, Goetsch, 1933, fue mal nominada de acuerdo al artículo 47.1 del International Code of Zoological Nomenclature (3); no existiría como taxon subordinado de Neotermes chilensis (Blanchard, 1851).

\section{BIBLIOGRAFIA}

(1) GOETSCH, W. 1933. Die chilenischen Termiten. Zoologische Jahrbücher 64:227-244.

(2) BLANCHARD, E. 1851. I Termianos. En: Gay, C. Historia Física y Política de Chile 6: 87-91. Imprenta de Maulde y Renou, París.

(3) RAMBUR, P. 1842. Histoire Naturelle des Insectes Névroptères. Librairie Encyclopédique de Roret. Paris. 529 p.

(4) VILLAN, L. 1972a. Estudio sistemático y biológico de Porotermes quadricollis (Rambur, 1842) (Insecta, Isoptera, Termopsidae, Porotermitinae) y métodos para determinar la resistencia termítica de la madera. Tesis para optar al título de Licenciado en Biología. Departamento de Zoología, Instituto Central de Biología, Universidad de Concepción. 182 p.

(5) VILLAN, L. 1972. El género Porotermes Hagen en Chile (Isoptera, Termopsidae). Boletín de la Sociedad de Biología de Concepción 44: 30-46.

(6) VILlAN, L. 1982. Descripción de las castas de Porotermes quadricollis (Rambur, 1842). Isoptera, Termopsidae, Porotermitinae. Boletín de la Sociedad de Biología de Concepción 53: 151-160.

(7) GUZMAN, R. 1966. Termes citados para Chile. Publicación Ocasional Museo Nacional de Historia Natural, Chile 10: 3-11.

(8) SNYDER, T.E. 1949. Catalog of the Termites (Isoptera) of the world. Smithsonian Miscellaneous Collections 112: $1-490$

(9) ARAUJO, R.L. 1977. Catálogo dos Isoptera do Novo Mundo. Ed. Academia Brasileira de Ciências. Río de Janeiro. 92 p.

(10) INTERNATIONAL CODE OF ZOOLOGICAL NOMENCLATURE. Ed. International Trust for Zoological Nomenclature. London. 1999. 306 p.

(11) KRISHNA, K. 1961. A generic revision and phylogenetic study of the Family Kalotermitidae (Isoptera). Bulletin of the American Museum of Natural History 122(4): 309-408.

(12) HAGEN, H. 1958. Linnaea Entomologica 12: 1-342.

(13) GRASSÉ, P.P., 1949. Traité de Zoologie, Paris, 9: 531.

Recibido: 28.04.04 Aceptado: 04.03.05 